\title{
STABLE EXPRESSION AND POTENTIAL USE OF WEST NILE VIRUS ENVELOPE GLYCOPROTEINS PREM/E AS ANTIGEN IN DIAGNOSTIC TESTS
}

\section{Juliana Felipetto Cargnelutti ${ }^{1}$, Mário Celso Sperotto Brum ${ }^{2}$, Rudi Weiblen ${ }^{1}$, Eduardo Furtado Flores ${ }^{1}$ *}

${ }^{1}$ Setor de Virologia, Departamento de Medicina Veterinária Preventiva, Centro de Ciências Rurais, Universidade Federal de Santa Maria, Santa Maria, RS, Brasil; ${ }^{2}$ Faculdade de Medicina Veterinária, Universidade Federal do Pampa, Uruguaiana, RS, Brasil.

Submitted: July 13, 2010; Returned to authors for corrections: December 01, 2010; Approved: January 31, 2011.

\begin{abstract}
West Nile virus (WNV) envelope glycoproteins preM/E were stably expressed in baby hamster kidney cells and tested as antigen in a fluorescent antibody assay for WNV antibodies. Sera from horses, mice and chicken immunized with an inactivated WNV vaccine and, less consistently, sera from horses acutely infected with WNV, reacted specifically with viral antigens present in preM/E-expressing cells.
\end{abstract}

Key words: West Nile virus, WNV, M/E glycoproteins, stable expression, diagnostic.

West Nile virus (WNV) is an emerging flavivirus maintained in nature through alternate cycles of infection in mosquitoes and wild birds, in which produces from subclinical infection to deadly disease (7). Occasionally, the virus is transmitted to humans, horses and other wild and domestic mammals, causing a self-limited febrile illness in mild cases, but severe meningitis or encephalitis in some cases (8). During decades, WNV infection was limited to North Africa, Middle East, Europe and Central Asia, causing sporadic, restricted outbreaks of human and/or animal disease $(7,16)$. In 1999, the virus was introduced in New York city (USA) by yet unknown means, causing around 60 human infections and seven deaths, and high mortality in wild and zoo birds (14). Following the initial outbreak, WNV progressively spread across the United States producing more than 25,000 human cases and around 1,200 deaths (7); near 30,000 horse cases and over 10,000 deaths (6). Extensive mortality among several bird species has also been documented during this outbreak (10). Moreover, WNV also spread northwards reaching Canada (17) and southwards, reaching Central America (12) the Caribean (11) and, Argentina $(1,13)$. In Brazil, there are no official reports of human or animal WNV infections up to date. However, the progressive spread of WNV southwards into South America has led to the concern of its possible introduction in Brazilian tropical and subtropical areas, where it would encounter favorable environmental conditions for maintenance and transmission (6). Thus, epidemiological surveillance in wild birds and horses - considered natural sentinels for WNV activity - holds critical towards early detection and adoption of measures to prevent or minimize human exposure upon the introduction of the virus.

As other flaviviruses, WNV contains a positive-sense linear RNA genome, wrapped inside an icosahedral protein coat covered externally by a lipid envelope (2). The lipid layer

*Corresponding Author. Mailing address: Setor de Virologia, Departamento de Medicina Veterinaria Preventiva, UFSM. Santa Maria, RS. 97105-900.; E-mail: eduardofurtadoflores@gmail.com 
of WNV particles contain the envelope (E) and membrane protein $(\mathrm{M})$, the later being synthesized as a precursor membrane (preM) protein in infected cells $(2,3,5)$. The $\mathrm{E}$ protein is the viral attachment protein, plays important roles in receptor binding, in fusion/penetration and contains most of the neutralizing epitopes (2,3). Thus, many gene-engineering flavivirus vaccines, including DNA vaccines, have been developed using the preM and E genes (4).

The gold standard serological assay for WNV is the plaque reduction neutralization test (PRNT) that is used as a confirmation and a titration technique for specific-WNV neutralizing antibodies. Immunoenzymatic tests (ELISAs) have also been used to detect WNV antibodies, and can be classified into three principal types: IgM antibody-capture ELISA, indirect ELISA to detect IgG and epitope blocking ELISA (4). Fluorescent antibody assays (IFA) performed over fixed WNV infected cells have also been used in some laboratories (4). In any case, antigens used in serological tests are either produced in cell culture or in mice (15). Thus, most procedures of antigen production for serologic tests involve virus manipulation. Nevertheless, the current WNV-free status would preclude manipulation of infectious virus in Brazilian territory, unless in special situations and under stringent laboratory containment. On the other hand, recombinant antigens expressed in drosophila cells, E. coli or mammalian cells have been shown to be adequate alternatives to be used in serological tests (4).

As to accommodate both needs, we used a strategy to produce WNV antigens for diagnosis without manipulating infectious virus. We herein describe the stable expression of WNV preM/E proteins in mammalian cells as an alternative for antigen production for WNV serological diagnosis.

Baby hamster kidney cells (BHK-21, ATCC, CCL-10) were lipofected with the plasmid vector pcWNME (kindly provided by Dr E. Konishi, Kobe University, Kobe, Japan). The vector pcWNME contains the coding region of WNV preM/E glycoproteins under the control of human cytomegalovirus (CMV) promoter (9). In addition to the expression cassette, the plasmid contains a selection marker for amplification in E.coli (ampicillin resistance) and a resistance marker for neomycin selection in eukaryotic cells (9). Plasmid amplification was conducted in E.coli and plasmidial DNA was purified using a plasmid DNA purification kit (Qiagen, Hilden, Germany). BHK-21 cells were cultivated in minimum essential medium (MEM), containing ampicillin (1.6mg/L), streptomycin $(0.4 \mathrm{mg} / \mathrm{L})$ and nistatin $(0.02 \mathrm{mg} / \mathrm{L})$, supplemented with $10 \%$ fetal bovine serum.

Forty eight hours after transfection, cells were trypsinized, submitted to limiting dilution in culture medium supplemented with $400 \mu \mathrm{g} / \mathrm{mL}$ of G-418 (Gibco-Invitrogen, Van Allen Way, Carlsbad, USA) and seeded on 96-well plates. G-418-resistant, single cell-derived colonies were observed after a few weeks of culture. Several resistant clones were selected and submitted to two additional cloning steps under G-418 selection. After three cycles of cloning, G-418-resistant clones were selected, amplified and investigated for expression of preM/E proteins by fluorescent antibody assay (IFA) as described below. After a first screening for protein expression, three clones expressing the proteins were selected for further characterization. The passage counting started after this step; cells were maintained in culture medium containing $400 \mu \mathrm{g} / \mathrm{mL}$ of G-418 during all passages and experiments. The selected clones were subcultured at every 4 to 6 days and protein expression was monitored by IFA at every two or three passages. The cells expressing WNV proteins will be thereafter designed BHK$\mathrm{M} / \mathrm{E}$.

The expression of WNV preM/E proteins in G-418resistant cell clones was routinely performed by indirect fluorescent antibody assay (IFA) performed on cell monolayers grown over glass coverslips. A flavivirus-specific mouse immune ascitis fluid (MIAF, provided by Dr Luiz Tadeu Moraes Figueiredo, Universidade de São Paulo, USP, Ribeirão Preto, SP, Brazil) was used as primary antibody and an antimouse FITC-conjugated as the secondary antibody (Sigma- 
Aldrich, St. Louis, MO, USA). Control BHK-21 cells were used as negative controls in all experiments and procedures.

Expression of WNV preM/E proteins in selected cell clones was detected by IFA up to cell passage 30 (Figure 1A). The percentage of positive cells (20-30\% to 50-60\%) and the intensity of the fluorescence varied over the cell passages (not shown). The reasons for these variations were not investigated, yet might result from some degree of toxicity of the expressed proteins for the transformed cells. No reaction was ever observed in BHK-21 cells, submitted to the same IFA protocol, confirming the specificity of the reaction (Figure 1B). Negative mouse serum incubated over fixed BHK-M/E and BHK-21 cells also yielded negative IFA results, attesting the specificity of the test (not shown). In addition, we performed an IFA using an anti-flavivirus monoclonal antibody (HB-112, ATCC) as the primary antibody. Again, positive IFA reaction was observed in BHK-M/E, but not in BHK-21 cells (Figure 1C, 1D). These results demonstrate that $\mathrm{BHK}-21$ cells transformed with the plasmid vector pcWNME and maintained under G-418 selection stable express WNV proteins preM/E. Recombinant proteins were recognized by a flavivirus-specific monoclonal antibody and by polyclonal mouse antisera (Figure 1A, 1C).

Next, we examined whether BHK-M/E cells would serve as antigen substrate in an IFA assay to detect anti-WNV antibodies in sera of different species. Glass slides containing acetone-fixed monolayers of BHK-M/E and control BHK-21 cells were incubated with undiluted sera of different sources, followed by washings in PBS and distilled water and incubation with the respective anti-species immunoglobulin FITC-conjugated (secondary antibodies were purchased from Sigma-Aldrich, St Louis, MO, USA). The origin of tested sera were, as follows: i. Sera from two horses vaccinated with a commercial inactivated WNV vaccine (West Nile Innovator, Fort Dodge Animal Health, Iowa, USA), collected at days 30 and 60 post-vaccination (pv); ii. Sera from four mice immunized with the same vaccine $(1 / 4$ of the recommended dose), collected at day $90 \mathrm{pv}$; iii. Serum samples from four vaccinated chicken ( $1 / 2$ of the dose, collected at day $60 \mathrm{pv})$; iv. Sera from two vaccinated rabbits (1/2 of the dose, days 30 and $60 \mathrm{pv})$. In addition, serum samples collected from 12 horses during acute, clinical WNV infection were also tested. These samples were positive for WNV antibodies in an IgM ELISA and were kindly provided by Dr Judith Wheeler, Veterinary Diagnostic Center, University of Nebraska at Lincoln, Lincoln, NE, USA. The results of IFA performed over fixed BHK-M/E and BHK-21 cells using these sera as primary antibody are shown in Table 1.

Sera from vaccinated horses (30 and 60 days pv) and mice (90 days pv) showed a frank positive reaction with BHK-M/E cells in the IFA assay (Table 1, Figure 1E, 1G). No fluorescence was observed in control BHK-21 cells incubated with these sera (Figure $1 \mathrm{~F}, 1 \mathrm{H}$ ), attesting the specificity of the reaction. Sera from vaccinated chicken (day $60 \mathrm{pv}$ ) reacted weakly with BHK-M/E cells, yet the reaction could be clearly distinguished from that observed in control cells (not shown). On the other hand, no specific fluorescent reaction was observed by using rabbit sera (days 30 and $60 \mathrm{pv}$ ) as primary antibody. These results show that sera of horses, mice and chickens (but not rabbits) immunized with an inactivated WNV vaccine contained antibodies (likely $\mathrm{IgG}$, as the secondary antibody used was anti-IgG) that specifically bound to WNV antigens expressed in BHK-M/E cells. The lack of reaction of immunized rabbit sera might be due to the low levels of antibodies induced by vaccination. On the other hand, the positive reaction by testing the other sera is promising towards the use of these cells as the antigen substrate in IFA for WNV antibodies. It is reasonable to speculate that natural WNV infection would induce higher antibody titers than an inactivated vaccine. Thus, is conceivable that convalescent sera from WNV naturally infected animals would contain enough antibodies as bind to viral antigens in BHK-M/E cells and yield positive fluorescence.

In order to further address the suitability of the BHK-M/Ebased IFA to detect WNV antibodies, sera obtained from 12 
horses during acute, clinical WNV infection were tested. These samples were previously tested positive for WNV IgM in a home-made ELISA (Judith Wheeler, VDC, UNL, personal communication). As shown in Table 1, four out of 12 samples reacted positively with viral antigens in BHK-M/E cells. Thus, sera from acutely affected, naturally infected horses also reacted with WNV antigens in IFA. It was not surprising that 8 out of 12 samples were negative in our test, since this test is designed to detect IgG. This class of immunoglobulin appears later than IgM during WNV infection, typically 10-12 days after the onset of clinical signs $(4,15)$. It is likely that sera collected at late stages of acute infection or after the convalescent period - when IgG reaches higher levels - would yield more consistent IFA positive results. In any case, as our test is intended to be used in serological surveys, it needs to be validated by testing a large number of WNV convalescent sera. Regardless, these preliminary results are promising towards the use of these antigens in serological tests.

The restriction for WNV manipulation only under stringent laboratory containment (BSL-3 facilities) has somehow delayed the production of reagents for serological diagnosis in WNV free-areas. Alternatives for antigen production include the use of viral vectors expressing WNV proteins and recombinant proteins produced in E.coli, insect and mammalian cells (4). The plasmid vector pcWNME - used in the present study - was constructed to investigate the immunogenicity of a DNA-based vaccine and its synergism with an inactivated vaccine (9). Transient expression of preM/E proteins was demonstrated in pcWNME-transfected BHK-21 cells and in mice immunized with the plasmid DNA (9). Nonetheless, stable protein expression by this vector has not been attempted or demonstrated. Then, before embarking on a bacterial or baculovirus expression system, we tried to express the recombinant proteins in BHK-21 cells in a stable fashion and investigated their suitability as antigen in a IFAbased serological assay. Recombinant proteins were detected for over 30 cell passages, being specifically recognized by antibodies present in the sera of WNV vaccinated animals. Moreover, antibodies of the sera of WNV acutely infected horses also bound specifically to recombinant proteins. Unfortunately, the unavailability of a large number of horse and avian sera positive for WNV antibodies precluded a more detailed examination on the specificity and sensitivity of the preM/E-based IFA assay, and whether it would be adequate for serological diagnosis in field samples. As the amount of protein expressed by transformed cells appears to be low, protein purification has not been attempted. Alternatives for abundant protein expression would include the use of bacterial or baculovirus-based expression systems to produce WNV antigens.

Summarizing, the stable expression of preM/E proteins in BHK-21 cells and their specific recognition by WNV antisera are promising towards their use as antigen in serological tests for WNV diagnosis and monitoring.

Table 1. Reactivity of sera from different animal species vaccinated or naturally infected with WNV, with BHK-ME cells and negative control (BHK-21 cells) by IFA.

\begin{tabular}{cccc}
\hline \multirow{2}{*}{ Origin } & \multirow{2}{*}{ Number of samples } & \multicolumn{2}{c}{ Positive reaction } \\
\cline { 3 - 4 } & 2 & BHK-M/E cells & BHK-21 cells \\
\hline Vaccinated horses & 4 & 2 & 0 \\
Vaccinated mice & 4 & 4 & 0 \\
Vaccinated chickens & 2 & $4 *$ & 0 \\
Vaccinated rabbits & 12 & 0 & 0 \\
Naturally infected horses & $4 * *$ & 0 \\
\hline
\end{tabular}

*Weak reaction, yet distinguishable from reaction on BHK-21 cells.

** Sera obtained during acute, clinical WNV infection. 


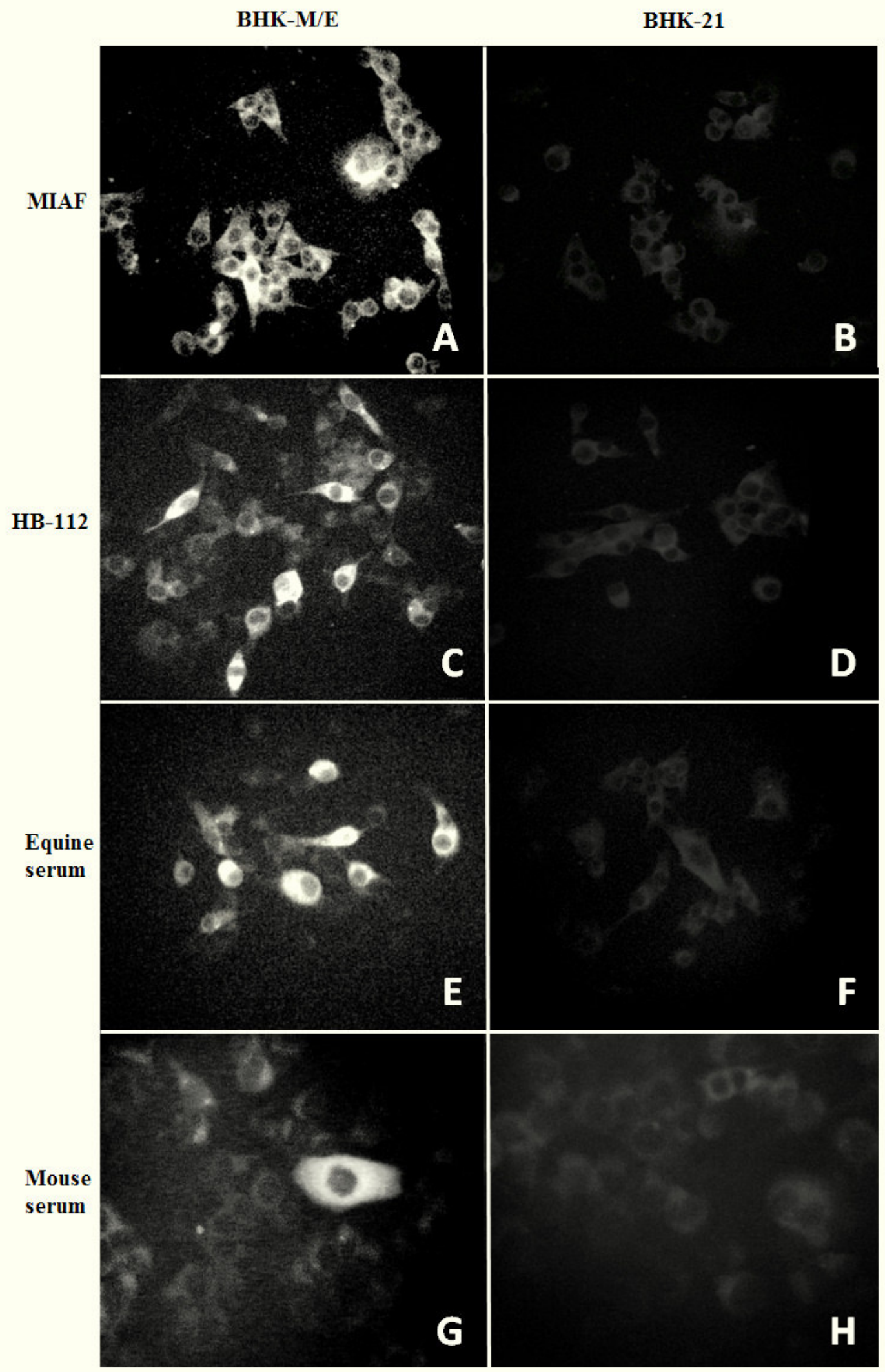

Figure 1. Reactivity of antibodies from different sources with WNV proteins preM and E expressed in BHK-21 cells, by immunofluorescence. Left column: BHK-M/E cells; right column: control BHK-21 cells. These cells were incubated with mouse ascitis fluid (MIAF) (A and B); flavivirus-specific monoclonal antibody HB-112 (C and D); serum from a horse immunized with a WNV inactivated vaccine $(\mathrm{E}$ and $\mathrm{F})$ and serum from a mouse immunized with the WNV vaccine $(\mathrm{G}$ and $\mathrm{H})$. Positive reaction was observed only in sera incubated with BHK-M/E cells. Photographs correspond to IFA performed on BHK-M/E cells at passages1 $\mathbf{1 6 5}$ (A); \# 22 (C) and \# 8 (E-G), and BHK-21 at passage \#35. 


\section{REFERENCES}

1. Adrian Diaz, L.; Komar, N.; Visintin, A.; Dantur Juri, M.J.; Stein, M.; Allende, R.J.; Spinsanti, L.; Konigheim, B.; Aguilar, J.; Laurito, M.; Almirón, W.; Contigiani, M. (2008). West Nile virus in birds, Argentina. Emerg. Infect. Dis. 14 (4), 689-691.

2. Brinton, M.A. (2002). The molecular biology of West Nile Virus: a new invader of the western hemisphere. Annu. Rev. Microbiol. 56, 371-402.

3. Chambers, T.J.; Hahn, C.S.; Galler, R.; Rice, C.M. (1990). Flavivirus genome organization, expression, and replication. Annu. Rev. Microbiol. 44, 649-88.

4. Dauphin, G.; Zientara, S. (2007). West Nile virus: recent trends in diagnosis and vaccine development. Vaccine. 25 (30), 5563-5576.

5. Diamond, M.S.; Pierson, T.C.; Fremont, D.H. (2008). The structural immunology of antibody protection against West Nile virus. Immunol. Rev. 225, 212-225.

6. Flores, E.F.; Weiblen, R. (2009). O vírus do Nilo Ocidental. Cienc. Rural 39 (2), 604-612.

7. Hayes, E.B.; Komar, N.; Nasci, R.S.; Montgomery, S.P.; O'Leary, D.R.; Campbell, G.L. (2005). Epidemiology and transmission dynamics of West Nile virus disease. Emerg. Infect. Dis. 11 (8): 1167-1173.

8. Hayes, E.B.; Sejvar, J.J.; Zaki, S.R.; Lanciotti, R.S.; Bode, A.V.; Campbell, G.L. (2005). Virology, pathology, and clinical manifestations of West Nile virus disease. Emerg. Infect. Dis. 11 (8), 1174-1179.

9. Ishikawa, T.; Takasaki, T.; Kurane, I.; Nukuzuma, S.; Kondo, T.; Konishi, E. (2007). Co-immunization with West Nile DNA and inactivated vaccines provides synergistic increases in their immunogenicities in mice. Microbes. Infect. 9 (9), 1089-1095.
10. LaDeau, S.L.; Kilpatrick, A.M.; Marra, P.P. (2007). West Nile virus emergence and large-scale declines of North American bird populations. Nature 447 (7145), 710-713.

11. Lefrancois, T.; Blitvich, B.J.; Pradel, J.; Molia, S.; Vachiery, N.; Martinez, D. (2006). West Nile virus in Guadeloupe: introduction, spread, and decrease in circulation level: 2002-2005. Ann. N. Y. Acad. Sci. 1081, 206-215.

12. Morales-Betoulle, M.E.; Morales, H.; Blitvich, B.J.; Powers, A.M.; Davis, E.A.; Klein, R.; Cordón-Rosales, C. (2006). West Nile virus in horses, Guatemala. Emerg. Infect. Dis. 12 (6), 1038-1039.

13. Morales, M.A.; Barrandeguy, M.; Fabbri, C.; Garcia, J.B.; Vissani, A.; Trono, K.; Gutierres, G.; Pigretti, S.; Menchaca, H.; Garrido, N.; Taylor, N.; Fernandez, F.; Levis, S.; Enría, D. (2006). West Nile virus isolation from equines in Argentina, (2006). Emerg. Infect. Dis. 12 (10), 15591561.

14. Nash, D.; Mostashari, F.; Fine, A.; Miller, J.; O'Leary, Huang, A.; D.; Murray, K.; Rosenberg, A.; Greenberg,, A.; Sherman, M.; Wong,, S.; Laynton, M. (2001). The outbreak of West Nile virus infection in the New York City area in 1999. N. Engl. J. Med. 344 (24), 1807-1814.

15. Tardei, G.; Ruta, S.; Chitu, V.; Rossi, C.; Tsai, T.F.; Cernescu, C. (2000). Evaluation of immunoglobulin M (IgM) and IgG enzyme immunoassays in serologic diagnosis of West Nile Virus infection. J. Clin. Microbiol. 38 (6), 2232-2239.

16. van der Meulen, K.M.; Pensaert, M.B.; Nauwynck, H.J. (2005). West Nile virus in the vertebrate world. Arch. Virol. 150 (4), 637-657.

17. Weese, J.S.; Baird, J.D.; DeLay, J.; Kenney, D.G.; Staempfli, H.R.; et al. (2003). West Nile virus encephalomyelitis in horses in Ontario: 28 cases. Can. Vet. J. 44 (6), 469-473. 